\title{
Relation between Geometric Dimensions of Coronary Artery Stenoses and Myocardial Perfusion Reserve in Man
}

\author{
Richard A. Goldstein, Richard L. Kirkeeide, Linda L. Demer, Michael Merhige, Akira Nishikawa, \\ Richard W. Smalling, Nizar A. Mullani, and K. Lance Gould \\ Cardiology Division, and Positron Diagnostic and Research Center, University of Texas \\ Health Science Center at Houston, Houston, Texas 77225
}

\begin{abstract}
To determine the relation between stenosis anatomy and perfusion in man, 31 patients had quantitative coronary arteriography and positron imaging (PET) with $\mathrm{Rb}-82$ or $\mathrm{N}-13$ ammonia at rest and after dipyridamole-handgrip stress. 10 patients were also studied after angioplasty (total stenoses $=41$ ). Percent narrowing and absolute cross-sectional luminal area were related through a quadratic function to myocardial perfusion reserve determined with PET. Arteriographically determined coronary flow reserve was linearly related to relative myocardial perfusion reserve as expected, based on the derivation of equations for stenosis flow reserve. All of the correlations had considerable scatter, indicating that no single measurement derived by coronary arteriography was a good indicator of perfusion reserve by PET in individual patients. This study provides the relation between all anatomic dimensions of coronary artery stenoses and myocardial perfusion reserve in man, and suggests that PET indicates the functional significance of coronary artery stenoses for clinical purposes.
\end{abstract}

\section{Introduction}

Coronary arteriography is the current "gold standard" for evaluating the significance of coronary artery lesions usually expressed in terms of percent diameter stenosis (\%D). ${ }^{1}$ However, this approach is limited, since other geometric characteristics of the stenosis such as the length and the absolute cross-sectional luminal area of the involved segment are not taken into account (1-4). Although quantitative coronary arteriography provides objective measures of all of these geometric dimensions, no one single anatomic measurement accurately predicts the severity of impaired perfusion. Consequently, Gould et al. proposed the

This paper was presented in part at the 1986 Society of Nuclear Medicine Annual Meeting, Washington, D.C., 24 June, 1986.

Address correspondence to Dr. Goldstein, Director, Nuclear Cardiology, Cardiology Div., The U. of Texas Medical School, P.O. Box 20708, Rm. 1.246, Houston, TX 77225. 1987.

Received for publication 7 August 1986 and in revised form 9 January

1. Abbreviations used in this paper: \%A, percent area stenosis; CFR, coronary flow reserve; \%D, percent diameter stenosis; $\mathrm{LAD}$, left anterior descending; LCX, left circumflex; Min A, minimal cross-sectional area (square millimeters); PET, positron emission tomography; RCA, right coronary artery; RMPR, relative myocardial perfusion reserve.

J. Clin. Invest.

(c) The American Society for Clinical Investigation, Inc.

$0021-9738 / 87 / 05 / 1473 / 06 \$ 1.00$

Volume 79, May 1987, 1473-1478 use of coronary flow reserve (CFR) as a physiologic measure of stenosis severity to integrate stenosis dimensions and fluid dynamic principles (5). This concept has been validated in animals with mechanical stenoses using electromagnetic flow meters on epicardial coronary arteries. Kirkeeide et al. developed the theoretical relation between stenosis dimensions and CFR, as well as validating it in dogs (4). However, these experimental studies did not address the relation between stenosis geometry and tissue perfusion, which may be affected by collaterals and changes in vascular space volume during high flow states; in addition, the relation of stenosis geometry to myocardial perfusion in man has not been previously demonstrated. Mullani has proposed a model relating perfusion and arterial flow reserve (6). Accordingly, the purpose of the current study was to determine the relation between anatomic measures of stenosis severity and regional myocardial perfusion reserve in man. All stenosis dimensions of length, absolute cross-sectional luminal area, and integrated length effects were determined by automated quantitative analysis of coronary arteriograms, and relative myocardial perfusion reserve (RMPR) was determined using positron emission tomography in conjunction with intravenous dipyridamolehandgrip stress in patients with coronary artery disease.

\section{Methods}

Patient population. 31 patients (19 men, 12 women) undergoing left heart catheterization and coronary arteriography for evaluation of chest pain, or undergoing an abnormal electrocardiographic stress test, made up the study population (Table I). In 10 of these patients, (seven men, three women), complete follow-up studies were also performed after percutaneous transluminal coronary angioplasty (total studies $=41$, interval between angioplasty and repeat positron scan $12.5 \pm 14.4 \mathrm{~d}$ ). The patients ranged in age from 33 to $70 \mathrm{yr}$ (mean $=51.9 \pm 10.0 \mathrm{yr}$ [SD]). None of the patients had left ventricular hypertrophy. Seven patients had a history of a previous transmural infarction. There were no patients with previous subendocardial infarction. All patients were imaged within 3 mo of their coronary arteriography and patients with intervening myocardial infarction or a change in angina were excluded from the study. The study protocol was approved by the University of Texas Medical School at Houston Committee for the Protection of Human Subjects, and all patients gave informed consent before entry into the study.

Coronary arteriography was performed using either the Judkins or Sones technique. Multiple coronary angiograms were obtained using 3$10 \mathrm{cc}$ of Hypaque-76 per injection with a biplane 5- or 7- or 9-in. image intensifier (Siemens AG, Erlanger, FRG). The x-ray tube was a model $150 / 40 / 72 \mathrm{c}$ with a $0.6-1.0-\mathrm{mm}$ focal spot operating at 4-6-ms exposures set at $300 \mathrm{~mA}$ and $109 \mathrm{kV}$. Low contrast Vari-Cath cine film was used at 30 to 60 frames per second. Orthogonal views were obtained for quantitative analysis of the angiograms. The cine system resolution was 4 to 5 line pairs per millimeter. Pincushion distortion and variation of magnification in different parts of the field were $<10 \%$ except at the corners of the field, but were corrected from measurements of an imaged grid whose dimensions were known (4).

Analysis of coronary angiograms. Stenoses of major proximal cor- 
Table I. Patient Characteristics

\begin{tabular}{|c|c|c|c|c|}
\hline Patient no. & Age & Sex & LVEF & MI \\
\hline 1 & 43 & $\mathbf{M}$ & 0.88 & - \\
\hline 2 & 36 & $\mathbf{M}$ & 0.65 & - \\
\hline 3 & 55 & $\mathrm{~F}$ & 0.53 & - \\
\hline 4 & 57 & $\mathbf{M}$ & 0.65 & - \\
\hline 5 & 50 & $F$ & 0.45 & + \\
\hline 6 & 62 & $\mathbf{M}$ & 0.42 & - \\
\hline 7 & 60 & $\mathbf{M}$ & 0.77 & - \\
\hline 8 & 48 & $F$ & 0.53 & + \\
\hline 9 & 37 & $\mathbf{M}$ & $n / a$ & - \\
\hline 10 & 61 & $\mathrm{~F}$ & 0.53 & + \\
\hline 11 & 55 & $\mathbf{M}$ & 0.69 & + \\
\hline 12 & 54 & $\mathbf{M}$ & 0.86 & - \\
\hline 13 & 34 & $\mathbf{M}$ & $n / a$ & - \\
\hline 14 & 49 & $F$ & 0.77 & - \\
\hline 15 & 49 & $\mathbf{F}$ & 0.62 & - \\
\hline 16 & 70 & $\mathbf{M}$ & 0.53 & + \\
\hline 17 & 50 & $\mathbf{M}$ & $n / a$ & - \\
\hline 18 & 43 & $\mathbf{M}$ & 0.83 & - \\
\hline 19 & 43 & $F$ & 0.77 & - \\
\hline 20 & 53 & $\mathbf{M}$ & 0.61 & - \\
\hline 21 & 47 & $\mathbf{M}$ & 0.71 & - \\
\hline 22 & 39 & $\mathbf{M}$ & 0.70 & - \\
\hline 23 & 60 & $\mathbf{M}$ & $\mathbf{n} / \mathbf{a}$ & - \\
\hline 24 & 51 & $F$ & 0.76 & - \\
\hline 25 & 61 & $\mathrm{~F}$ & 0.71 & - \\
\hline 26 & 33 & $\mathbf{M}$ & 0.74 & - \\
\hline 27 & 58 & $F$ & 0.55 & - \\
\hline 28 & 60 & $\mathrm{~F}$ & 0.83 & - \\
\hline 29 & 67 & $\mathrm{~F}$ & 0.67 & + \\
\hline 30 & 66 & $\mathbf{M}$ & 0.51 & + \\
\hline 31 & 59 & $\mathbf{M}$ & 0.76 & - \\
\hline
\end{tabular}

F, Female; LVEF, left ventricular ejection fraction; M, male; MI, history of previous myocardial infarction; $n / a$, not available.

onary arteries were quantitatively analyzed as previously described (4). Briefly, end-diastolic frames from orthogonally paired angiographic views of each stenosis were optically magnified, digitized, and corrected for the point spread function of the $\mathrm{x}$-ray system, and provided a final spatial resolution of $\pm 0.1 \mathrm{~mm}$. After selecting the arterial segment of interest, the images were analyzed by an automated arterial border recognition program and cross-sectional densitometry from which vessel diameters and cross-sectional areas were determined. Spatial calibration was determined using the catheter as a size reference. Specific anatomic characteristics of each stenosis, including minimal cross-sectional area, relative percent narrowing (diameter and area), and integrated length effects were determined. Dimensional measurements by this method have been previously shown to be accurate to within $\pm 0.1 \mathrm{~mm}$ in x-ray phantoms of coronary arteries $(0.5-5.0 \mathrm{~mm}$ in diameter) in scattering media, and reproducible to within 2 to $3 \%(7,8)$.

Pressure-flow characteristics of the stenotic segment were then derived by computing the viscous and expansion coefficients of pressure loss that were used to determine the coronary stenosis flow reserve. CFR was taken as the positive root of the following equation:

$\left(C e Q n^{2}\right) C F R^{2}+\left(C v Q n+\frac{P a-P v}{C F R_{n}}\right) C F R-(P a-P v)=0$,

where $C v$ and $C e$ are constants related to the pressure losses occurring along the stenosis and determined by its X-ray geometry, $P a$ and $P v$ are the aortic and venous pressures, $Q n$ is the normal resting coronary flow rate, and $C F R_{n}$ is the normal CFR. The following values were assumed: $C F R_{n}=5 ; P a=100 \mathrm{mmHg} ; P v=10 \mathrm{mmHg}$; and $Q n=$ product of normal cross-sectional lumen area multiplied by $15 \mathrm{~cm} / \mathrm{s}$ (the assumed normal resting flow velocity). This approach has been previously validated over a range of $1-4 \pm 0.5$ times normal resting flow (4). In this paper, percent narrowing and CFR were reported as the most severe stenosis from orthogonal views.

Positron imaging protocol. Patients were fasted for at least $4 \mathrm{~h}$ before the study and drinks or drugs containing theophylline or caffeine were withheld for at least $8 \mathrm{~h}$ to avoid antagonizing the vasodilatory effects of dipyridamole. Chest fluoroscopy was performed to mark the inferior border of the heart and patients were then positioned in the University of Texas positron camera. A transmission scan (200-million counts) was performed using a plexiglass ring containing $3 \mathrm{mCi}$ of $\mathrm{Ga}-68$. The ring was then removed, the patient's position rechecked, and a resting emission scan was obtained by intravenous injection of either $R b-82(n=7$, mean dose $38.9 \pm 7.7 \mathrm{mCi}$ at rest and again at stress) or $\mathrm{N}-13$ ammonia $(n=34$, mean dose $17.1 \pm 1.35 \mathrm{mCi}$ at rest, $16.9 \pm 1.74 \mathrm{mCi}$ at stress) in an ungated mode without time-of-flight correction as previously described (9).

For $\mathrm{Rb}-82$, acquisition of emission images was started $1 \mathrm{~min}$ after the end of infusion to minimize blood pool activity. Data acquisition was stopped 5-7 min later. For $\mathrm{N}-13$ ammonia, acquisition of images was started $3 \mathrm{~min}$ after injection and continued for $15 \mathrm{~min}$. After an appropriate delay to allow for decay of counts from the resting study, the same tracer was injected during stress induced by dipyridamole plus handgrip stress (Table II). Patients were given a $0.142-\mathrm{mg} / \mathrm{kg}$ per min intravenous infusion of dipyridamole for $4 \mathrm{~min}$ (total dose, 0.568 $\mathrm{mg} / \mathrm{kg}$ ). The intravenous line was then flushed with normal saline and electrocardiograms were recorded. $4 \mathrm{~min}$ after the dipyridamole infusion was completed, handgrip was performed by the patient using a mechanical spring device at $25 \%$ of maximal strength and maintained for $4 \mathrm{~min} .2$ min into handgrip, the tracer was injected and emission images were acquired in a manner identical to the resting study. Particular attention was paid to positioning of the patient in the same location as in the rest scan. All patients were able to complete the protocol.

Analysis of positron images. Nine transaxial slices of the rest and stress studies were displayed in color on a computer remote terminal monitor. Regions of interest in the lateral, anterior, septal, and posterior left ventricular walls were defined using an interactive software program that recorded the mean activity, number of pixels, and standard deviation of the activity. A histogram of the activity in each region of interest was also obtained to minimize inclusion of admixtures of normal and abnormal tissue. Mean activity in each region had a standard deviation under $10 \%$ of mean activity for that region. If a stress defect was present, it was quantified for each slice on which it was evident. Regions with resting defects or regional wall motion abnormalities were not included in the analyses.

Average activity for each region was calculated for resting and stress images. RMPR by positron tomography was calculated by the following equation: RMPR = (S/R stenosis)/(S/R normal), where $S$ and $R$ represent regional activity during stress (dipyridamole plus handgrip) and rest, respectively. This value, therefore, represents the fraction of normal myocardial perfusion reserve within the region supplied by the stenotic artery. Although not an absolute measure of perfusion reserve per se, this approach avoids the necessity of obtaining arterial samples to measure

Table II. Intravenous Dipyridamole-Handgrip Stress Protocol

\begin{tabular}{ll}
\hline Time & Intervention \\
\hline $\min$ & \\
$0-4$ & Intravenous dipyridamole $(0.142 \mathrm{mg} / \mathrm{kg}$ per $\mathrm{min})$ \\
$5-6$ & Flush lines, electrocardiogram obtained \\
$6-10$ & Handgrip at $25 \%$ maximum \\
8 & Inject tracer \\
\hline
\end{tabular}


the arterial input function of the tracer. In the event that more than one defect was present, the defect corresponding to the most severe stenosis was used for analysis and the normal reference region was verified from the arteriogram.

Statistical analysis. The relation between RMPR and quantitative arteriographic measures of stenosis severity were determined using a linear or quadratic best fit to a least-squares equation.

\section{Results}

The artery and segment used for quantitative analysis is listed in Table III. Of the 41 coronary artery stenoses analyzed, there were 25 left anterior descending (LAD) coronary arteries (15 proximal, 10 middle), 8 left circumflex (LCX) coronary arteries ( 3 proximal, 5 middle), 6 right coronary arteries (RCA) (1 proximal, 3 middle, and 2 distal), and single stenoses of the middle ramus and middle diagonal coronary arteries. 10 patients of the 31 studied had double vessel disease, and one patient had triple vessel disease (Table IV).

Relation between coronary anatomy and RMPR. Coronary stenoses with a percent diameter narrowing of $50 \%$ or greater were associated with a reduction in RMPR (Fig. 1). As the stenosis became tighter RMPR fell, but there was considerable scatter from the mean curve relating the two parameters. The relation

Table III. Quantitative Coronary Arteriographic and RMPR Data

\begin{tabular}{|c|c|c|c|c|c|c|c|c|}
\hline Patient no. & Artery & Segment & $\% \mathrm{D}$ & $\% A$ & $\operatorname{Min} A$ & CFR & Coll & RMPR \\
\hline $1 \mathrm{~A}$ & LAD & Middle & 68 & 90 & 0.60 & 1.7 & - & 0.70 \\
\hline B & & & 51 & 73 & 1.70 & 3.4 & & 0.86 \\
\hline 2 & LAD & Middle & 55 & 80 & 1.70 & 3.0 & + & 0.90 \\
\hline 3 & Ramus & Middle & 73 & 86 & 0.83 & 2.2 & - & 0.81 \\
\hline 4 & LAD & Proximal & 77 & 99 & 0.19 & 0.6 & - & 0.70 \\
\hline 5 & RCA & Middle & 70 & 94 & 0.53 & 1.0 & + & 0.60 \\
\hline $6 \mathrm{~A}$ & LAD & Proximal & 66 & 94 & 0.90 & 1.2 & - & 0.86 \\
\hline B & & & 54 & 73 & 2.70 & 3.7 & & 1.00 \\
\hline 7 & RCA & Middle & 77 & 91 & 0.80 & 1.5 & + & 0.62 \\
\hline 8 & LCX & Middle & 66 & 80 & 1.38 & 3.0 & - & 0.76 \\
\hline 9 & LAD & Middle & 40 & 80 & 1.84 & 3.1 & - & 0.94 \\
\hline 10 & RCA & Proximal & 54 & 80 & 3.57 & 2.8 & - & 0.92 \\
\hline 11 & LAD & Middle & 65 & 87 & 0.71 & 2.0 & + & 0.88 \\
\hline $12 \mathrm{~A}$ & LAD & Proximal & 78 & 95 & 0.40 & 0.9 & - & 0.77 \\
\hline B & & & 59 & 77 & 2.10 & 2.6 & & 0.95 \\
\hline $13 \mathrm{~A}$ & LAD & Proximal & 74 & 90 & 0.50 & 1.8 & - & 0.75 \\
\hline B & & & 21 & 62 & 3.00 & 4.3 & & 1.00 \\
\hline $14 \mathrm{~A}$ & LAD & Proximal & 74 & 91 & 0.30 & 1.4 & - & 0.74 \\
\hline B & & & 53 & 67 & 1.00 & 4.0 & & 1.00 \\
\hline $15 \mathrm{~A}$ & LAD & Middle & 67 & 75 & 0.60 & 3.4 & - & 0.89 \\
\hline B & & & 62 & 77 & 0.90 & 3.2 & & 0.80 \\
\hline 16 & LCX & Middle & 47 & 66 & 2.10 & 3.9 & + & 0.89 \\
\hline $17 \mathrm{~A}$ & LCX & Proximal & 78 & 93 & 0.60 & 1.2 & - & 0.76 \\
\hline B & & & 55 & 74 & 2.40 & 3.5 & & 0.93 \\
\hline 18 & LCX & Middle & 62 & 82 & 1.36 & 2.8 & - & 0.94 \\
\hline 19 & LAD & Middle & 42 & 56 & 1.36 & 4.2 & - & 1.00 \\
\hline $20 \mathrm{~A}$ & LCX & Middle & 78 & 95 & 0.40 & 0.9 & - & 0.79 \\
\hline B & & & 80 & 96 & 0.40 & 0.7 & & 0.79 \\
\hline 21 & RCA & Distal & 59 & 89 & 0.25 & 1.6 & - & 0.89 \\
\hline 22 & RCA & Distal & 52 & 67 & 1.52 & 3.8 & - & 1.00 \\
\hline $23 \mathrm{~A}$ & LAD & Proximal & 69 & 89 & 1.09 & 1.6 & - & 0.82 \\
\hline B & & & 70 & 77 & 1.50 & 3.1 & & 0.96 \\
\hline 24 & LAD & Proximal & 44 & 63 & 3.84 & 4.0 & - & 1.00 \\
\hline 25 & LAD & Proximal & 51 & 71 & 2.50 & 3.9 & - & 0.87 \\
\hline 26 & LAD & Middle & 64 & 84 & 1.25 & 2.6 & - & 0.88 \\
\hline 27 & LCX & Proximal & 38 & 59 & 3.26 & 4.4 & - & 1.00 \\
\hline $28 \mathrm{~A}$ & LAD & Proximal & 62 & 87 & 0.90 & 2.2 & - & 0.91 \\
\hline B & & & 50 & 72 & 1.30 & 2.5 & & 0.97 \\
\hline 29 & RCA & Middle & 60 & 46 & 3.86 & 4.4 & - & 1.00 \\
\hline 30 & LAD & Middle & 32 & 59 & 4.79 & 4.5 & + & 0.99 \\
\hline 31 & DIAG & Middle & 56 & 72 & 1.23 & 3.5 & + & 1.00 \\
\hline
\end{tabular}

A, preangioplasty; Min A, minimal cross-sectional lumen area (millimeters); B, postangioplasty study; Coll, presence of collaterals; DIAG, diagonal coronary artery; LAD, LAD coronary artery; LCX, LCX coronary artery. The artery listed represents data from the most severe stenosis. Segment refers to the region of the artery analyzed. 
Table IV. Additional Quantitative Coronary Angiographic Data on Patients with Multivessel Coronary Disease

\begin{tabular}{lllrrll}
\hline Patient no. & Artery & Segment & \%D & \%A & Min A & CFR \\
\hline 3 & LCX & Middle & 55 & 94 & 0.90 & 2.9 \\
4 & LCX & Proximal & 78 & 91 & 0.46 & 0.9 \\
& DIAG & Proximal & 68 & 90 & 0.58 & 1.6 \\
5 & LAD & Proximal & 54 & 85 & 2.08 & 2.5 \\
6 & LCX & Proximal & 53 & 82 & 2.51 & 2.9 \\
7 & LAD & Proximal & 50 & 71 & 4.23 & 3.7 \\
11 & RCA & Middle & 100 & 100 & - & - \\
13 & LCX & Middle & 55 & 79 & 2.83 & 3.1 \\
16 & RCA & Middle & 100 & 100 & - & - \\
21 & LCX & Proximal & 46 & 55 & 3.94 & 4.4 \\
23 & LCX & Proximal & 43 & 73 & 0.80 & 4.1 \\
24 & RCA & Proximal & 100 & 100 & - & -
\end{tabular}

DIAG, diagonal coronary artery; LAD, LAD coronary artery; LCX, LCX coronary artery.

between RMPR and percent area stenosis (\%A) was similar (Fig. 2). RMPR was near normal until the luminal area reduction approached $70 \%$ or greater, and then decreased. Fig. $3 \mathrm{~A}$ plots the relation of absolute minimal cross-sectional area of the stenosis and RMPR for all 41 studies analyzed. A minimal crosssectional area of $>3.0 \mathrm{~mm}^{2}$ was associated with normal perfusion reserve, whereas values lower than this area did not allow separation of normal from abnormal perfusion reserve. Part of the scatter observed is probably related to expected differences in normal lumen size for different arteries and segments. In Fig. 3 $B$, minimal cross-sectional lumen area from the 15 studies of proximal LAD coronary arteries is plotted against relative perfusion reserve with a much better fit than for all artery segments $(r=0.84)$. The relations between relative perfusion reserve and absolute or relative percent narrowing were flat, and then sharply curvilinear beyond a certain advanced degree of anatomic severity. In contrast, CFR correlated linearly with RMPR by positron imaging (Fig. 4). Table $\mathrm{V}$ lists the regression equations of anatomy versus relative perfusion reserve and $r$ values for the entire data set and the largest subset, proximal LAD coronary stenoses. In general, the greatest improvement in curve fitting was for minimal cross-sectional luminal area and the least for CFR. These data suggest that the precise arterial segment is required to relate minimal cross-sectional area to perfusion,

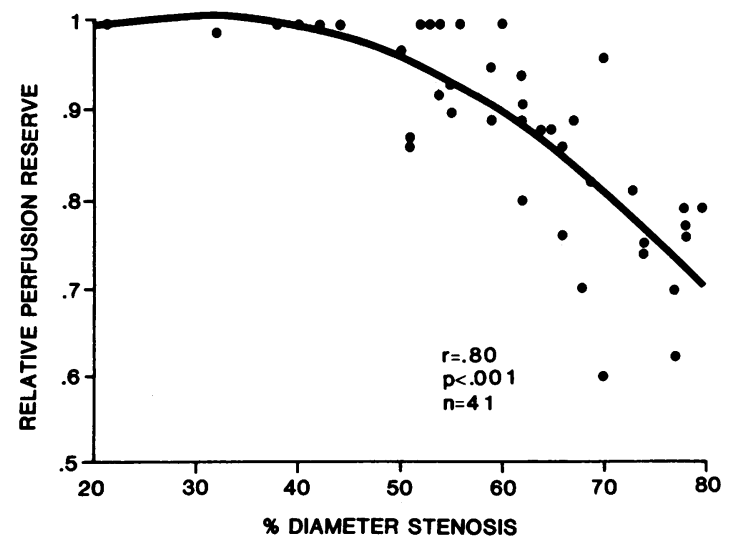

Figure 1. Relation of RMPR and \%D.

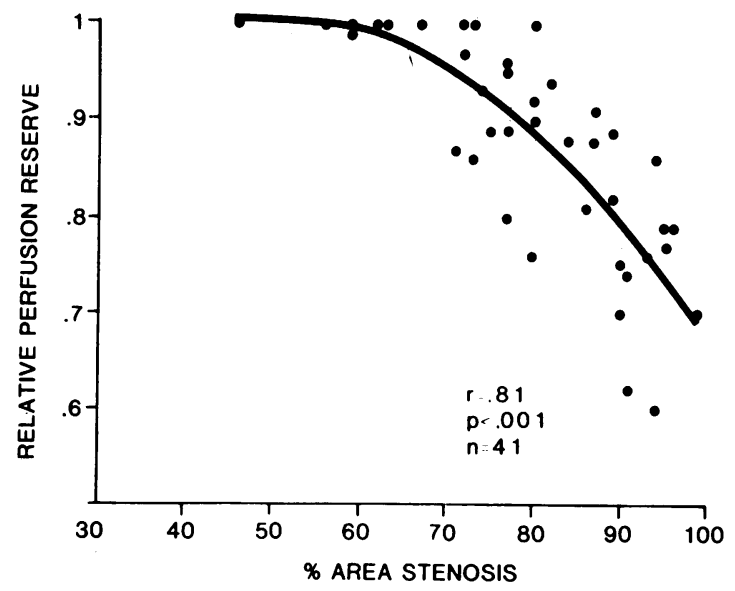

Figure 2. Relation of RMPR and \%A.

whereas CFR is a more generally applicable measure of perfusion, since it includes all stenosis dimensions in its estimate.

\section{Discussion}

Until the current study, the relation between geometric dimensions of coronary artery stenoses and myocardial perfusion reserve had not been determined in man. The results indicate that both $\% \mathrm{D}$ and $\% \mathrm{~A}$ are important determinants of regional myo-
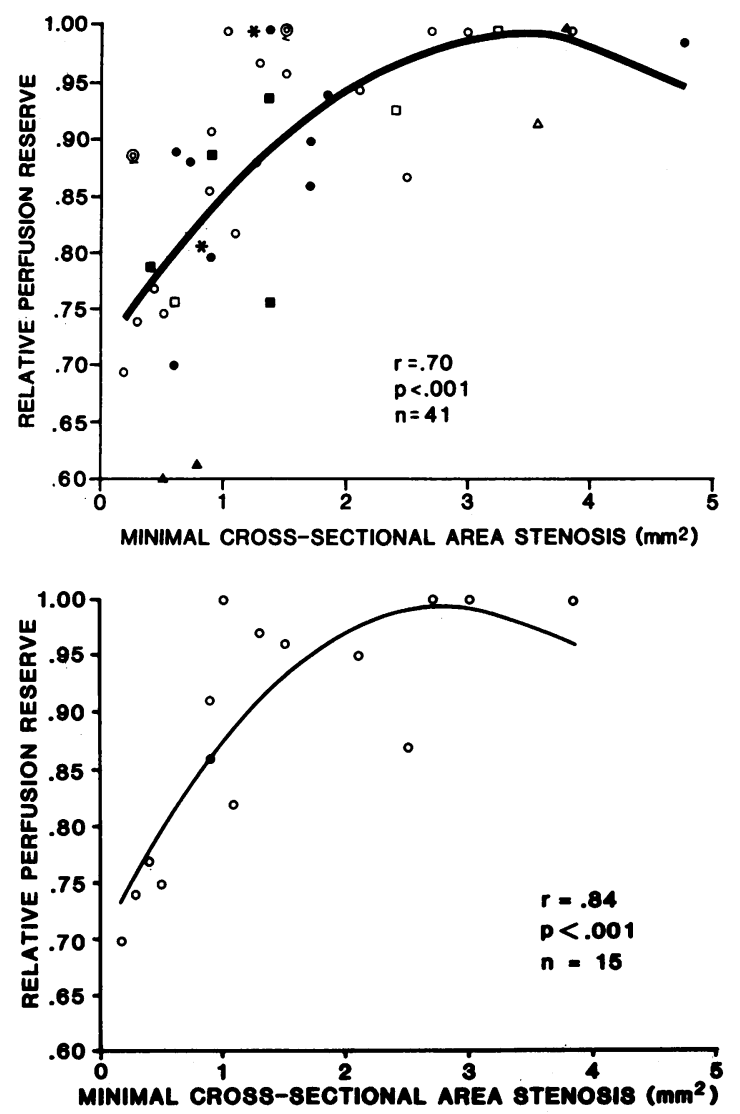

Figure 3. (A) Relation of RMPR and absolute minimal cross-sectional area. (O) Proximal LAD; (๑) middle LAD; ( $\square$ ) proximal LCX; ( $)$ middle LCX; $(\Delta)$ proximal RCA; $(\Delta)$ middle RCA; (๑) distal RCA; ( $)$ middle other. $(B)$ Relation of RMPR and absolute minimal cross-sectional area in proximal LAD stenoses. 


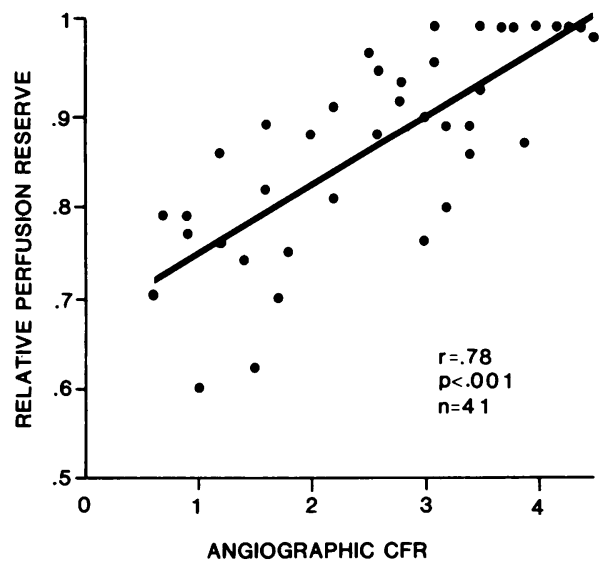

Figure 4. Relation of RMPR and CFR derived from quantitative coronary arteriography.

cardial perfusion reserve. Myocardial perfusion reserve was, on the average, normal for vessels with less than a 50\% diam narrowing or less than a $70 \%$ area narrowing (Figs. 1 and 2). However, since the curve relating percent narrowing to perfusion reserve falls off rapidly as stenosis severity increases beyond these critical values, percent narrowing alone does not readily indicate the physiologic significance of a stenosis. The nonlinearity of the relation of percent narrowing to myocardial perfusion reserve is consistent with the fact that flow is inversely related to the radius of the vessel to the fourth power $(10,11)$.

Although absolute minimal cross-sectional area also correlated with RMPR ( $r=0.70, P<0.001)$, it was a poor predictor

Table V. Correlation of Quantitative Angiographic Data and RMPR

\begin{tabular}{|c|c|c|}
\hline Parameters compared & Regression equations & $r$ value \\
\hline \multicolumn{3}{|l|}{ \% D vs. RMPR } \\
\hline LAD proximal & $\begin{array}{c}0.842+0.010 \mathrm{x}-0.0002 \mathrm{x}^{2} \\
( \pm 0.141) \quad( \pm 0.005) \quad\left( \pm 0.0 \times 10^{-5}\right)\end{array}$ & 0.87 \\
\hline All & $\begin{array}{c}0.900+0.007 x-0.0012 x^{2} \\
( \pm 0.139) \quad( \pm 0.005) \quad\left( \pm 4.5 \times 10^{-5}\right)\end{array}$ & 0.80 \\
\hline \multicolumn{3}{|l|}{ \% A vs. RMPR } \\
\hline LAD proximal & $\begin{array}{c}0.499+0.018 x-0.0002 x^{2} \\
( \pm 0.785) \quad( \pm 0.020) \quad( \pm 0.0001)\end{array}$ & 0.89 \\
\hline All & $\begin{array}{c}0.664+0.014 \mathrm{x}-0.0001 \mathrm{x}^{2} \\
( \pm 0.332) \quad( \pm 0.009) \quad\left( \pm 5.8 \times 10^{-5}\right)\end{array}$ & 0.81 \\
\hline \multicolumn{3}{|l|}{ Min A vs. RMPR } \\
\hline LAD proximal & $\begin{array}{c}0.698+0.211 x-0.037 x^{2} \\
( \pm 0.044) \quad( \pm 0.059) \quad( \pm 0.015)\end{array}$ & 0.84 \\
\hline All & $\begin{array}{c}0.712+0.165 x-0.024 x^{2} \\
( \pm 0.034) \quad( \pm 0.040) \quad( \pm 0.009)\end{array}$ & 0.70 \\
\hline \multicolumn{3}{|l|}{ CRF vs. RMPR } \\
\hline LAD proximal & $\begin{array}{cc}0.704 & +0.073 x \\
( \pm 0.036) & ( \pm 0.013)\end{array}$ & 0.84 \\
\hline All & $\begin{array}{cc}0.675 & +0.075 x \\
( \pm 0.028) & ( \pm 0.010)\end{array}$ & 0.78 \\
\hline
\end{tabular}

Groups listed represent patients with proximal stenoses of the LAD coronary artery (LAD proximal, $n=15$ ) and data from all studies $(n=41)$. The values in parentheses are the standard errors of the estimates. CFR, angiographic CFR. of impaired perfusion reserve when applied to all 41 studies. However, the relation was improved $(r=0.84)$ when only proximal LAD lesions were compared to relative perfusion reserve (Fig. $3 \mathrm{~B}$ ). These results are similar to data from Harrison et al. who reported abnormal coronary flow (velocity) reserve with a suction Doppler probe in patients during open heart surgery when the minimal cross-sectional area of the LAD coronary artery was $<4.5 \mathrm{~mm}^{2}$ (3). However, they concluded that absolute cross-sectional luminal area was a good indicator of stenosis severity $(r=0.69, P<0.005)$ compared with percent narrowing, which appeared to be independent of flow reserve. The apparent discrepancy between the two studies is probably due to differences in the severity of stenoses present in each study.

Most of Harrison's cases had a \%D $<50 \%$ and an absolute minimal cross-sectional area $>3.0 \mathrm{~mm}^{2}$. In contrast, our group had a higher prevalence of more severe stenoses (34 of 41 had a $>50 \%$ diam stenosis, with only 6 of 41 having a minimal cross-sectional area $>3.0 \mathrm{~mm}^{2}$ ). Of the proximal LAD stenoses, 13 of 15 had a $>50 \%$ diam stenosis, and only 2 of 15 had a minimal cross-sectional area of $3.0 \mathrm{~mm}^{2}$ or greater. Consequently, our data covers a greater range of severities more appropriate for making a general case. Another difference between the two studies is the fact that the majority of patients included in Harrison's study had diffuse three vessel coronary disease that may have been undetected by coronary arteriography, whereas in the current study most of the patients had single vessel disease.

A reason why percent narrowing and absolute cross-sectional area may not be closely related to perfusion reserve may be due to the use of a normal arterial segment as a reference. For example, percent narrowing of a diffusely diseased artery may underestimate the severity of a lesion, since the stenosis diameter cannot be referenced to a normal segment. Under these conditions, minimal cross-sectional area would better correlate with impaired flow. However, in the absence of diffuse disease, percent narrowing would be a better marker of stenosis severity, since it relates the cross-sectional luminal area to the normal segment. Similarly, the physiologic significance of a given absolute crosssectional lumen area depends on what the normal lumen size was before narrowing occurred. A $1-\mathrm{mm}^{2}$ cross-sectional lumen area of an artery that had a normal lumen area of $2 \mathrm{~mm}^{2}$ is not a severe stenosis, whereas the same $1-\mathrm{mm}^{2}$ luminal area in an artery that was originally $8 \mathrm{~mm}^{2}$ is a severe lesion. This variation between segmental and diffuse disease in different-sized arteries in patient groups is part of the reason why no single geometric dimension alone can be used as a general approach to determine stenosis severity in most patients.

The correlation between myocardial perfusion reserve and arteriographically determined coronary stenosis flow reserve, integrating all stenosis dimensions, was linear. These results agree with animal studies demonstrating a close relation between arteriographic coronary stenosis flow reserve and arterial flow reserve directly measured using electromagnetic flow probes (4). However, in patients, the effects of collaterals, perfusion pressure, vasomotor tone, and changes in the size of the vascular bed during coronary arteriolar vasodilation are potentially confounding problems adding to data scatter, but that are accounted for by direct perfusion imaging with positron emission tomography.

\section{Limitations of the study}

It is important to examine potential limitations in the assessment of perfusion with positron emission tomography. Positron- 
emitting radiotracers have decay characteristics that permit quantification of regional myocardial activity in vivo (12). Regional myocardial perfusion can be estimated using $\mathrm{N}-13$ ammonia or Rb-82 by direct measurement of tracer uptake (1316). Schelbert et al. have previously demonstrated that uptake of $\mathrm{N}-13$ ammonia is linearly related to perfusion determined by radioactive microspheres from 0.5 to 3.0 times normal resting flow (13). However, at higher flow rates, ammonia uptake plateaus and therefore underestimates perfusion. This plateauing of uptake is due to a fall in extraction fraction at high flows.

A decreased extraction fraction during hyperemia produces similar effects on $\mathrm{Rb}-82$ uptake in relation to perfusion (15-17). This underestimation of true flow at high flows probably results in stenoses having to be more severe before a reduction in RMPR is observed by positron imaging (PET). Consequently, if quantitative flows could be measured by correcting for the reduced extraction at high flows, even milder, and therefore earlier, stenoses could be identified. Other limitations of positron imaging include partial volume errors and cardiac motion (12). In this study, RMPR was determined at rest and after dipyridamolehandgrip stress using the same regions of interest, an approach that minimizes these potential sources of error.

Another potential source of error on the assessment of regional myocardial perfusion reserve is in patients with proximal three-vessel disease. The tomographic images available for analysis consisted of 4 to 6 slices that encompass the entire myocardium. Each slice contained an anterior, lateral, and septal region, and on distal slices a posterior region as well. In all patients studied thus far, there has been at least one region supplied by a normal proximal vessel. However, in a patient with proximal stenoses of all three coronary arteries, the measurement of RMPR by positron imaging may be in error. Since the majority of the patients had single-vessel coronary disease, additional studies are necessary before this approach can be extended to patients with multivessel disease.

\section{Implications}

Coronary arteriography is an important part of the evaluation of patients with coronary artery disease, but only provides indirect measures of myocardial perfusion. No single anatomic measure of stenosis severity obtained by quantitative coronary arteriography accurately predicts perfusion reserve. RMPR by positron tomography indicates the physiologic significance of stenoses noninvasively. With the expected economical availability of Rb-82 in the absence of a cyclotron, noninvasive assessment of the functional severity of coronary artery disease will be possible.

\section{Acknowledgments}

The authors wish to acknowledge the contribution of Mary Haynie, R.N., Richard Holmes, Salma Marani, Mark Franceschini, Leonard A. Bolomey, Ross Hartz, Dan Burgess, Mark Wilson, Wai-Hoi Wong, Ph.D., Timothy J. Tewson, Ph.D., Mark Berhige, Ph.D., and the technical staff of the Hermann Hospital cardiac catheterization laboratory, without whom this study would not have been possible. We also appreciate the secretarial help of Shirley Mickelsen.

This work was carried out as a joint collaborative research project with the Clayton Foundation for Research, and was supported in part by grants R01-HL-26862 and R01-HL-26855 from National Institutes of Health, grant DE-FG05-84ER60210 from the Department of Energy, and by a Grant-in-Aid from the American Heart Association.

\section{References}

1. Seeley, B. D., and D. F. Young. 1976. Effect of geometry on pressure losses across models of arteroid stenoses. J. Biomech. 9:439-438.

2. Gould, K. L. 1978. Noninvasive assessment of coronary stenoses by myocardial imaging during coronary vasodilation. I. Physiologic principles and experimental validation. Am. J. Cardiol. 41:267-278.

3. Harrison, D. G., C. W. White, L. F. Hiratzka, D. B. Doty, D. H. Barnes, C. L. Eastham, and K. L. Marcus. 1984. The value of lesion cross-sectional area determined by quantitative coronary angiography in assessing the physiologic significance of proximal left anterior descending coronary arterial stenoses. Circulation. 69:1111-1119.

4. Kirkeeide, R. L., K. L. Gould, and L. Parsel. 1986. Assessment of coronary stenoses by myocardial perfusion imaging during pharmacologic coronary vasodilation. VII. Validation of coronary flow reserve as a single integrated functional measure of stenosis severity reflecting all its geometric dimensions. J. Am. Coll. Cardiol. 7:103-113.

5. Gould, K. L., K. Lipscomb, and G. W. Hamilton. 1974. Physiologic basis for assessing critical coronary stenosis. Am. J. Cardiol 33:87-94.

6. Mullani, N. A. 1984. Myocardial perfusion with rubidium-82. III Theory relating severity of coronary stenosis to perfusion deficit. $\mathrm{J}$. Nucl. Med. 25:1190-1196.

7. Gould, K. L., K. O. Kelley, and E. L. Bolson. 1982. Experimental validation of quantitative coronary arteriography for determining pressure-flow characteristics of coronary stenoses. Circulation. 66:930-937.

8. Kirkeeide, R. L., P. Fung, R. W. Smalling, and K. L. Gould. 1982. Automated evaluation of vessel diameter from arteriograms. Proc. IEEE Comp. Soc., Comp. Cardiol., New York. 215-218.

9. Gould, K. L., R. A. Goldstein, N. Mullani, R. L. Kirkeeide, W.-H. Wong, T. J. Tewson, M. S. Berridge, L. A. Bolomey, R. K. Hartz, R. W. Smalling, F. Fuentes, and A. Nishikawa. 1986. Noninvasive assessment of coronary stenoses by myocardial perfusion imaging during pharmacologic coronary vasodilation. VIII. Clinical feasibility of positron cardiac imaging without a cyclotron using generator-produced Rubidium82. J. Am. Coll. Cardiol. 7:775-789.

10. Poiseuille, J. L. M. 1846. Recherches experimentales sur le mouvement des liquides dans les tables tres petite diametres. Mem. Acad. Sci. (Paris). 9:433.

11. Gould, K. L., and K. Lipscomb. 1974. Effects of coronary stenoses on coronary flow reserve and resistance. Am. J. Cardiol. 34:48-55.

12. Goldstein, R. A., N. A. Mullani, and K. L. Gould. 1983. Quantitative myocardial imaging with positron emitters. Progress in Cardiology, Vol. 12. P. N. Yu and J. F. Goodwin, editors. Lea \& Febiger, Philadelphia. 147-191.

13. Schelbert, H. R., M. E. Phelps, E. J. Hoffman, S. C. Huang, C. E. Selin, and D. E. Kuhl. 1979. Regional myocardial perfusion assessed with $\mathrm{N}-13$ labeled ammonia and positron emission computerized axial tomography. Am. J. Cardiol. 43:209-218.

14. Shah, A., H. R. Schelbert, M. Schwaiger, E. Henze, H. Hansen, C. Selin, and S. C. Huang. 1985. Measurement of regional myocardial blood flow with $\mathrm{N}-13$ ammonia and positron emission tomography in intact dogs. J. Am. Coll. Cardiol. 5:92-100.

15. Mullani, N. A., R. A. Goldstein, K. L. Gould, D. J. Fisher, S. K. Marani, and H. A. O'Brien. 1983. Myocardial perfusion with Rubidium82. I. Measurement of extraction fraction and flow with external detectors. J. Nucl. Med. 24:898-906.

16. Goldstein, R. A., N. A. Mullani, D. J. Fisher, S. K. Marani, K. L. Gould, and H. A. O'Brien. 1983. Myocardial perfusion with Rubidium-82. II. The effects of metabolic and pharmacologic interventions. J. Nucl. Med. 24:907-915.

17. Goldstein, R. A. 1985. Kinetics of Rubidium-82 after coronary occlusion and reperfusion: assessment of patency and viability in openchested dogs. J. Clin. Invest. 75:1131-1137. 